\title{
The Influence of Magnetic Field on Synthesis of Iron Nanoparticles
}

\author{
Ualkhanova $\mathrm{M}^{1}$, Perekos $\mathrm{AY}^{2}$, Dubovoy $\mathrm{AG}^{3}$, Schur $\mathrm{DV}^{3 *}$, Al D Zolotarenko ${ }^{3}$, An D Zolotarenko ${ }^{3}$, \\ Gavrylyuk NA ${ }^{3}$, Gabdullin $\mathrm{MT}^{1,4}$, Ramazanov TS ${ }^{1}$, Akhanova $\mathrm{N}^{1,4}$ and Orazbayev $\mathrm{S}^{1}$ \\ ${ }^{1}$ Al-Farabi Kazakh National University, Al-Farabi 71, Almaty \\ ${ }^{2}$ Institute of Metal Physics NAS of Ukraine, 36 Vernadsky Ave., Kiev \\ ${ }^{3}$ Institute of Problems of Materials Science, NAS of Ukraine, St. Krzhizhanovskogo 3, Kiev \\ ${ }^{4}$ Kazakhstan-British Technical University, Tole bi 59, Almaty
}

*Corresponding author: Schur DV, Institute of Problems of Materials Science, NAS of Ukraine, St. Krzhizhanovskogo 3, Kiev, 03142 Ukraine, Tel: +380974452982, E-mail: dmitry.schur@gmail.com; marjan_0309@mail.ru

Citation: Ualkhanova M, Perekos AY, Dubovoy AG, Schur DV, Al D Zolotarenko, et al (2019) The Influence of Magnetic Field on Synthesis of Iron Nanoparticles. J Nanosci Nanotechnol Appl 3: 302

Article history: Received: 30 September 2019, Accepted: 30 October 2019, Published: 04 November 2019

\begin{abstract}
One of today's tasks is the development of new strategies for the synthesis of ferromagnetic, superparamagnetic nanoparticles immobilized in a polymer or carbon nanostructured matrix, and studying of the possibility of influencing their structure and magnetic properties.

When the critical parameter that determines certain phenomena (the size of magnetic domains, the characteristic length of exchange interactions, the length of magnetostatic interactions, etc.) becomes comparable to the particle size, nanoscale magnetic materials exhibit sharp (dramatic) variability of magnetic properties. Therefore, there is a need to develop new methods of synthesis, protection and certification of such nanosystems to synthesize promising magnetic materials using technologically controlled processes.

The encapsulation of metal nanoparticles into carbon chemically inert graphite like shells allows the synthesis of new generation materials to begin. Magnetic nanoparticles occupy an important place among these materials and such nanoparticles, which can be technologically important magnetic materials, will be investigated in this paper.

The formation of nanocomposites, the production of nanoparticles of the same size and, accordingly, with reproducible properties, is one of the main technological tasks of this research. Methods of obtaining, which allow us to precisely control the size of particles are continuously improved. Magnetic nanostructured materials with unique electrical, mechanical, catalytic, optical and, moreover, specific magnetic properties, make it possible to constantly expand their fields of application in computer science, catalysis, for sensors production, in medicine and in biology.

The correlation between nanostructure and magnetic properties allows us to offer different types of magnetic nanostructures: (1) systems with isolated particles, whose unique magnetic properties derive from decreasing sizes of components; (2) ultrafine particles with a cortical structure; (3) materials in which magnetic interaction is the dominant property; (4) nanocomposites, which consist of magnetic particles encapsulated in a chemically inert matrix. The magnetic properties in this case are determined by the ratio of volume fractions of magnetic particles and the matrix.

In this work, we studied the effect of an external magnetic field on the formation and phase composition of iron particles synthesized in an arc discharge in a liquid phase. These products can be technologically important magnetic materials.
\end{abstract}

Keywords: Carbon Chemically Inert Graphite like Shells; Nanoparticles; Magnetic Field, Synthesis; Structure

\section{Introduction}

Recently, nanoparticles (NPs) and nanostructures (NSs) have attracted close attention of researchers for their unique properties, potential applications, including new types of very active and selective catalysts, chemical and biological sensors, drug components, high-density media, magnet and optoelectronic devices, etc [1]. Most of the unusual properties of NPs are due to their high ratio of surface area to volume. The ability to control the value of specific surface of NP and its chemical composition opens up the possibility of controlling their properties and functional features. Surface phenomena were mainly studied in thin films and, to a lesser extent, in nanoparticles and materials based on nanoparticles.

The formation of nanocomposites, the production of particles of the same size and reproducible properties, is the main technological task for today. The search for synthesis methods that allow us to precisely control the particle size is continuous. Some methods that make it possible to obtain composites of polymers with nanoparticles already exist. 
When the critical parameter that determines certain phenomena (the size of magnetic domains, the characteristic length of exchange interactions, the length of magnetostatic interactions, etc.) becomes comparable to particles size, nanoscale magnetic materials exhibit a sharp (dramatic) change in magnetic properties. Therefore, there is a need to develop new strategies for the synthesis and certification of such systems to produce promising magnetic nanomaterials in a controlled manner.

The correlation between nanostructure and magnetic properties allows us to offer various types of magnetic nanostructures [2]: (I) systems with isolated particles, whose unique magnetic properties result from the decreasing sizes of the components; (II) ultra-thin particles with a cortical structure; (III) nanocrystalline materials, in which a substantial part of the volume is grain boundaries and intergranular spaces (interfaces) materials, in which magnetic interactions are the dominant property; (IV) nanocomposites consisting of magnetic particles directed onto a chemically inert matrix. The magnetic properties in this case are determined by the ratio of volume fractions of magnetic particles and the matrix.

The strategy of preparing composites containing ferromagnetic nanoparticles should be based on methods that allow not only to obtain stable products with high yield, but also the ability to control the particles size, their size distribution and interparticle intervals, etc. For the production of magnetic nanocomposites, such methods are widely used as the introduction of metal ions into a polymer matrix, the metallization of polymer surface, the evaporation of metal atoms, the ultrasonic method, a homogeneous molecular assembly, etc.

For example, nanocomposites containing $\mathrm{Fe}_{3} \mathrm{O}_{4}$ nanoparticles and $\mathrm{g}-\mathrm{Fe}_{2} \mathrm{O}_{3}(£ 20 \mathrm{~nm}$ ) were obtained by the exchange reaction of iron salts with perfluorin on ion-mine membranes (Nafion) by alkaline hydrolysis [3]. Ultrasonic decomposition of Fe(CO) was carried out in the presence of various surface-active substances, including polyvinylamine [4]. Covered $\mathrm{Fe}_{2} \mathrm{O}_{3}$ nanoparticles had a diameter of 5-16 nm. In another method [5], $\mathrm{C}^{2+}$ ions adsorbed in an ion exchange resin were reduced by excess $\mathrm{NaBH}_{4}$. Nanocomposites of a Co-B alloy with a wide particle size distribution (3-30 nm) were obtained.

The same approach was used to reduce cobalt salts in a solution of polyvinylpyridine [6]. The coercive force of a cobalt-containing polystyrene nanocomposite after low-temperature annealing (up to $720 \mathrm{~K}$ ) grew up to 5 times [7].

According to another method, carbonyl $\mathrm{Co}_{2}(\mathrm{CO})_{8}$, embedded in the matrix of copolymer-styrene with DFA or isopropanol solution, can also be obtained after annealing at $470 \mathrm{~K}$. Spherical cobalt nanoparticles with an average diameter of $26 \mathrm{~nm}$ were obtained by this method [8]. Magnetic properties were also found in Ni-polyethylene and Co-polyethylene nanocomposites. The conditions for their preparation were also given [9]. Although the methods of synthesis of such nanocomposites are well known, most of them have drawbacks such as a wide and shapeless particle size distribution, difficult to control composition and crystallinity of multicomponent systems.

Therefore, the novelty of this work is in studying of self-organized metal-carbon nanoparticles, in which the synthesis of ferromagnetic nanoparticles and nanocarbon matrix occurs simultaneously in an external magnetic field.

It has already been proven that the composition of ferromagnetic nanoparticles, their size (5-30 nm) and size distribution as well as the thickness of a stabilizing polymer shell can be controlled at the stage of nanocomposite synthesis [10-15]. Previous studies have shown that nanocomposites of this type exhibit ferromagnetic properties at room temperature, with the coercive force depending on the pretreatment conditions. By changing the synthesis conditions (temperature, the ratio of the output reagents, the nature of the stabilizing matrix), nanoparticles of elemental metals, their oxides and carbides of the required size, shape and structure (for example, the inner shell) and with a uniform distribution in the matrix can be obtained [12]. It can be controlled in this way, and magnetic properties can be changed. In the case of bimetallic nanohybrid compositions (Fe-Pt, Fe-Co), the possibility of obtaining intermetallic compounds on a nano-scale was analyzed.

Magnetic nanoparticles for studying various limiting phenomena, including magnetic coupling through a nonmagnetic layer and anisotropic exchange effects ( $\mathrm{Co}-\mathrm{CoO}-\mathrm{Co}$, $\mathrm{Co}-\mathrm{Pd}-\mathrm{Co}$ compounds, $\mathrm{Co}-\mathrm{TiO}_{2}-\mathrm{Co}$ compounds), magnetic proximity effects ( $\mathrm{Co}-\mathrm{Pd}, \mathrm{Fe}-\mathrm{Pd}$ ), exchange-jump effects ( $\mathrm{SmCo}-\mathrm{Co}, \mathrm{Nd}-\mathrm{Fe}-\mathrm{B}-\mathrm{Fe} \mathrm{B}_{3} \mathrm{~B}$ ) were also prepared by thermal decomposition of metal-containing compounds in a liquid (mineral oil or polymer solution in oil) [16-17].

Magnetic recording is the predominant technology for data storage, and further promises a rapid progressive increase in recording density. The goal of magnetic production to achieve a recording density of 1 Tbit / in 2 requires a further reduction in the size of magnetic particles to about $4 \mathrm{~nm}$ with an anisotropy that is strong enough not to change their magnetic moments during thermal vibrations. Particular attention was paid to Pt alloys with $3 \mathrm{~d}$ metals for their high magnetocrystalline anisotropy. Alternative ways of synthesizing highly anisotropic isolated, self-organizing magnetic nanoparticles [18] and their magnetic stability [19] are currently under investigation.

One of the methods for producing highly dispersed ferromagnetic nanoparticles is the arc discharge method in the liquid phase (DLF) [20-21]. This method has a number of technological and physical advantages. It is quite simple in hardware design, has environmental cleanliness, waste-free technology, low energy consumption, economical. In addition, this method is characterized by high temperatures $(\sim 4000 \mathrm{~K})$ in the zone of nanoparticle synthesis, ultra-high cooling rates $(\sim 109 \mathrm{~K} / \mathrm{s})$, and high dispersion of the resulting product $(10-1000 \mathrm{~nm})$. 
The DLF method consists in the excitation of an electric discharge between two electrodes or pieces of material to be dispersed, placed in a dielectric liquid medium. Under the influence of a spark discharge, the microregions of electrodes or granules are melted and metallic vapor and molten droplets are released into the surrounding liquid (spark discharge zone), in which metal particles are formed during their rapid cooling.

With DLF method in area of plasma channel and in the surrounding liquid, electric and magnetic fields of complex configuration occur, which obviously influence the dispersion process. In this connection, the purpose of this work was to study the influence of an external constant magnetic field on the phase composition and dispersion of iron nanoparticles.

\section{Experimental Details}

DLF were subjected to electrodes from steel 45 , containing $0.66 \%$ by weight C according to X-ray elemental analysis oxygen and carbon atoms) on a device designed by authors of [21]. The schematic diagram of the installation is shown in Figure 1. The voltage between the electrodes was $300 \mathrm{~V}$, the average current during the spark discharge was in the range of 200-300 mA. The crystal structure and phase composition of the composite was studied using a DRON-3.0 X-ray diffractometer in cobalt radiation, and the magnetic properties were studied using a ballistic magnetometer in the field range up to $800 \mathrm{kA} / \mathrm{m}$ at room temperature. An external magnetic field of $80 \mathrm{kA} / \mathrm{m}$ was formed using a permanent magnet, between the poles of which a reactor for the synthesis of powders was placed. Particle sizes were determined in three ways: by broadening lines in electron diffraction patterns (fine fraction), by electron micrographs (middle fraction), and by broadening lines on radiographs (large fraction) by the approximation method [22].

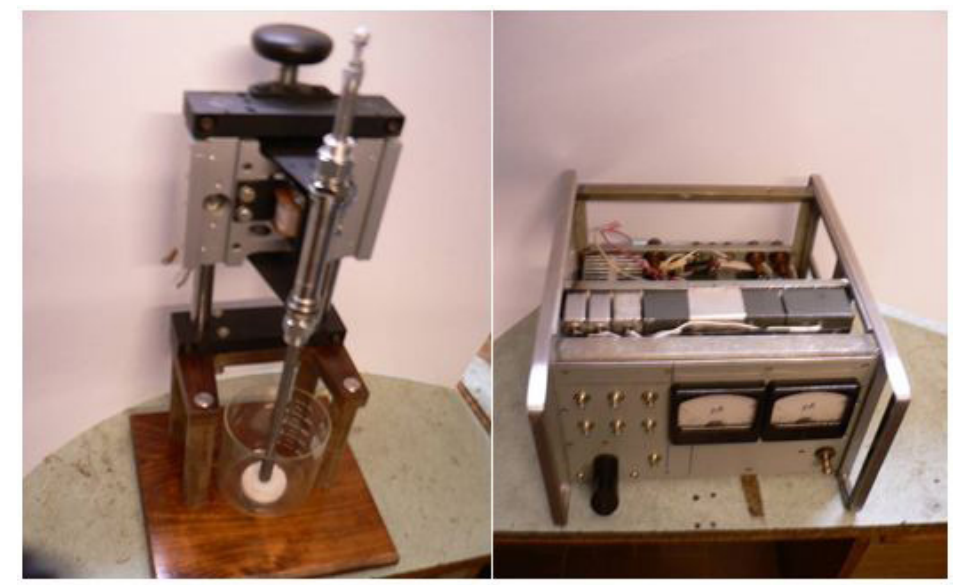

Figure 1: Installation for the synthesis of nanocarbon structures and Me-carbon composites in the liquid phase with an electromagnetic vibrator

\section{Results and Discussion}

Figures 2 and 3 show electron diffraction patterns and micrographs of highly dispersed Fe particles, obtained in distilled water and ethanol without a magnetic field and during its application. Micrographs show spherical particles with a wide particle size distribution. Using these micrographs, it is easy to determine the size of particles of the middle fraction, which, according to Table 1, are in the range of 200-300nm. The sizes of particles of the smallest fraction can be determined from the broadening of lines in the electron diffraction patterns, and the sizes of the particles of the largest fraction. That makes the dominant contribution to the X-ray scattering, from the broadening of the X-ray lines. The results of these calculations are also given in Table 1, and it indicates that the sizes of particles obtained by the DLF method are in a wide range of sizes from $20 \mathrm{~nm}$ to $1 \mu \mathrm{m}$. This conclusion is consistent with the results of studies by other authors, obtained in a number of works [20-21, 23-25, 26-33]. The experimental results shown in Figures 2 and 3, as well as in Table 1, indicate that the application of a magnetic field has a noticeable effect on the dispersion of particles of only a small fraction, leaving the sizes of particles of medium and coarse fractions almost unchanged.

\begin{tabular}{|c|c|c|c|c|c|}
\hline \multirow{2}{*}{$\begin{array}{c}\text { Conditions of } \\
\text { receipt }\end{array}$} & \multicolumn{2}{|c|}{ Phase composition } & \multicolumn{3}{|c|}{ Particle size, $\mathrm{nm}$} \\
\hline & phases & $\%$ & EG & TEM & X-ray SA \\
\hline $\begin{array}{l}\text { Water, } \\
\mathbf{H}=\mathbf{0}\end{array}$ & $\begin{array}{l}a-\mathrm{Fe} \\
\mathrm{Fe}_{3} \mathrm{O}_{4}\end{array}$ & $\begin{array}{c}95 \\
5\end{array}$ & 90 & 300 & 1000 \\
\hline $\begin{array}{l}\text { Water, } \\
\mathbf{H} \neq \mathbf{0}\end{array}$ & $\begin{array}{l}\alpha-\mathrm{Fe}, \\
\mathrm{Fe}_{3} \mathrm{O}_{4}\end{array}$ & $\begin{array}{c}96 \\
4 \\
\end{array}$ & 60 & 300 & 1000 \\
\hline $\begin{array}{c}\text { Ethanol, } \\
\text { H }=0\end{array}$ & $\begin{array}{l}\alpha-\mathrm{Fe} \\
\gamma-\mathrm{Fe}\end{array}$ & $\begin{array}{c}93 \\
7\end{array}$ & 30 & 200 & 180 \\
\hline $\begin{array}{c}\text { Ethanol, } \\
\mathbf{H} \neq \mathbf{0}\end{array}$ & $\begin{array}{l}\alpha-\mathrm{Fe} \\
\gamma-\mathrm{Fe}\end{array}$ & $\begin{array}{c}95 \\
5\end{array}$ & 20 & 200 & 150 \\
\hline
\end{tabular}




\begin{tabular}{|c|c|c|c|c|c|}
\hline \multirow{2}{*}{$\begin{array}{c}\text { Conditions of } \\
\text { receipt }\end{array}$} & \multicolumn{2}{|c|}{ Phase composition } & \multicolumn{3}{|c|}{ Particle size, nm } \\
\cline { 2 - 6 } & phases & $\%$ & EG & TEM & X-ray SA \\
\hline $\begin{array}{c}\text { Glycerol, } \\
\text { H=0 }\end{array}$ & $\alpha-\mathrm{Fe}$ & 100 & - & - & 90 \\
\hline $\begin{array}{c}\text { Glycerol, } \\
\mathbf{H} \neq \mathbf{0}\end{array}$ & $\begin{array}{c}\alpha-\mathrm{Fe}, \\
\text { С (графит) }\end{array}$ & $\begin{array}{c}90 \\
10\end{array}$ & - & - & 90 \\
\hline $\begin{array}{c}\text { Toluene, } \\
\mathbf{H}=\mathbf{0}\end{array}$ & $\begin{array}{c}\alpha-\mathrm{Fe}, \gamma- \\
\mathrm{Fe}, \mathrm{Fe}_{3} \mathrm{C}\end{array}$ & $\begin{array}{c}20,30 \\
50\end{array}$ & - & - & $-, 30,80$ \\
\hline $\begin{array}{c}\text { Toluene, } \\
\mathbf{H} \neq \mathbf{0}\end{array}$ & $\begin{array}{c}\gamma-\mathrm{Fe}, \\
\mathrm{Fe}_{3} \mathrm{C}\end{array}$ & $\begin{array}{c}30 \\
70\end{array}$ & - & - & 25,30 \\
\hline
\end{tabular}

Table 1: Phase composition and particle sizes of iron powders obtained in distilled water, ethanol, glycerin, toluene when an external magnetic field is applied
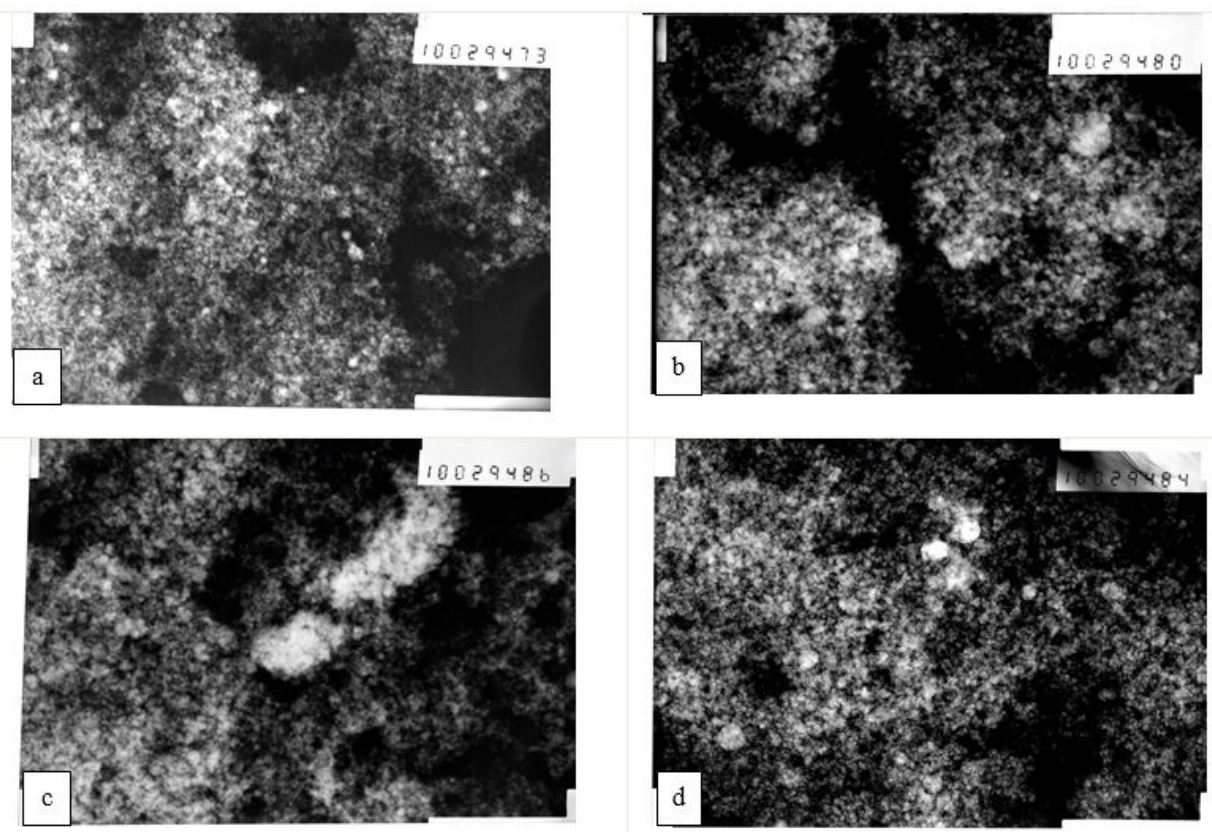

Figure 2: Electron micrographs of iron powders obtained in distilled water ( $a, b)$ and ethanol (c, d) without a magnetic field (a, c) and when it is applied (b, d)

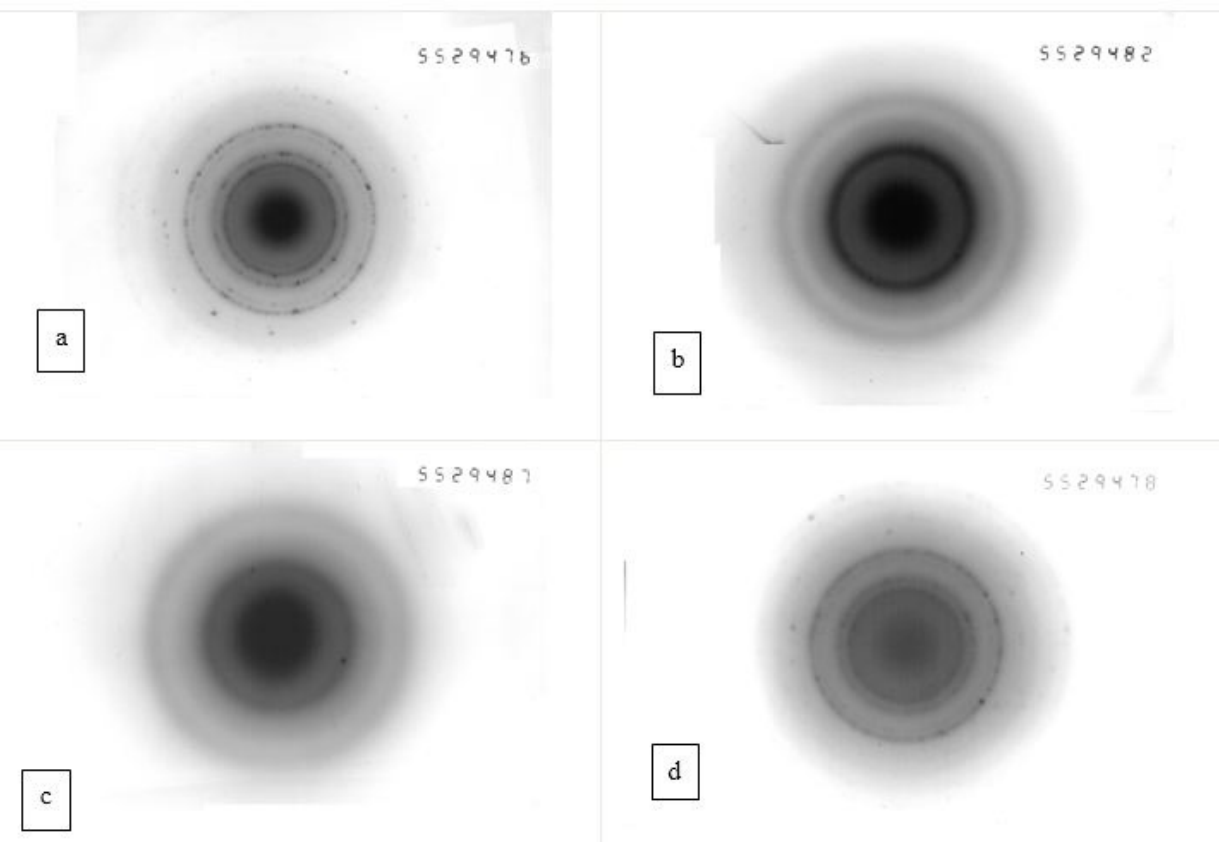

Figure 3: Electronic microdiffraction of iron powders obtained in distilled water $(a, b)$ and ethanol (c, d) without a magnetic field (a, c) and during its application (b, d) 
Figures 4 and 5 shows the X-ray diffractograms of all investigated samples, both without overlapping and with the application of a magnetic field, and Table 1 shows the results of determining their phase composition. These results indicate that the phase composition of the product obtained in various liquids, when a magnetic field is applied, behaves differently. Therefore, the phase composition of iron nanoparticles obtained in distilled water and ethanol varies only in terms of the percentage of observed phase components, the amount of less magnetic $\mathrm{Fe}_{3} \mathrm{O}_{4}$ oxide and $\gamma$-Fe when a magnetic field is applied, a few $(\sim 1$ and $2 \%$, respectively) decrease. For iron nanoparticles obtained in glycerol and toluene, both the quality and the number of observed phases change: graphite appears in glycerol when a magnetic field is applied, and the strong magnetic phase disappears in toluene $\alpha$-Fe.

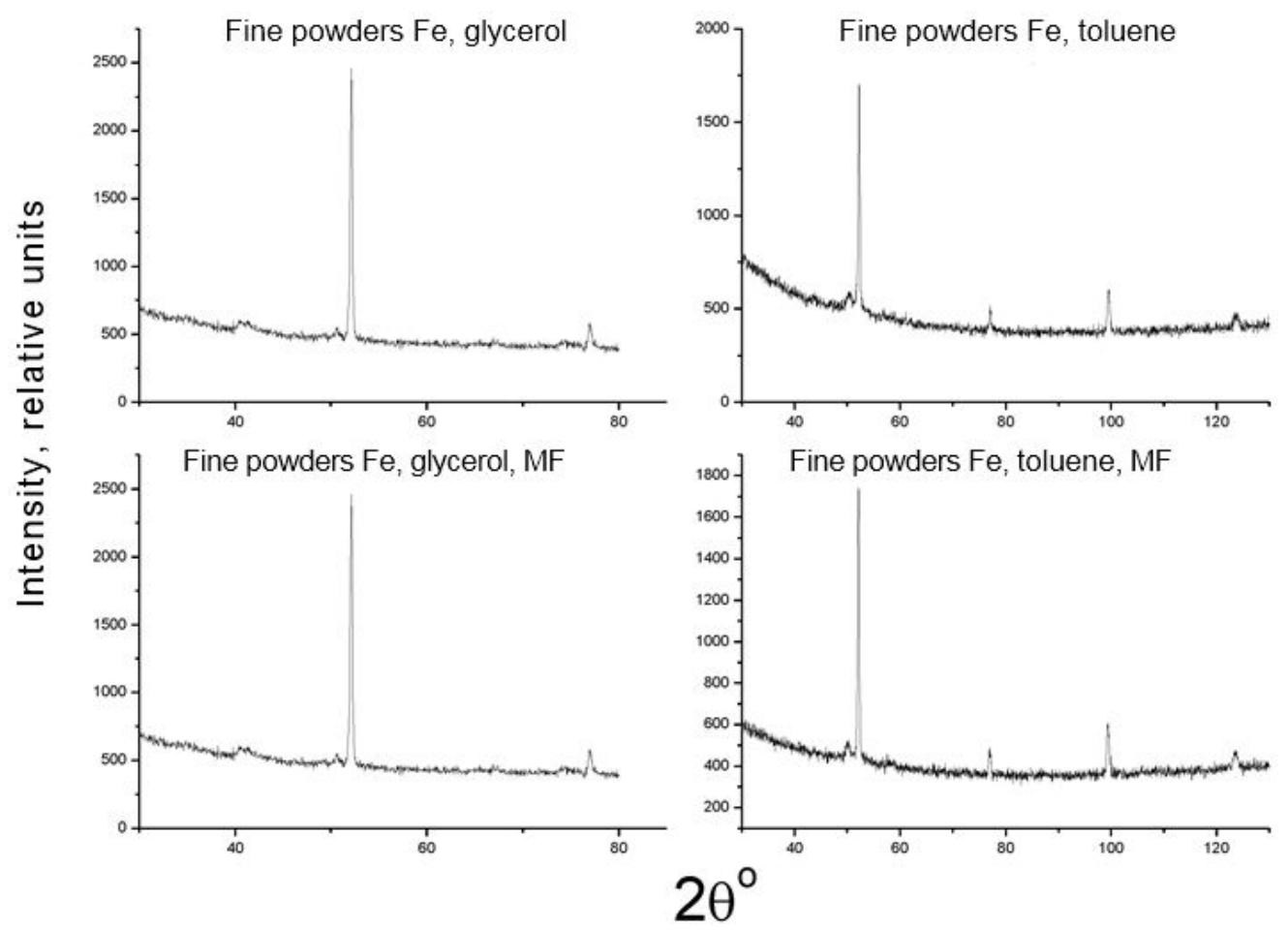

Figure 4: Fragments of diffractograms of iron nanoparticles obtained in water and ethanol without MF and during its application
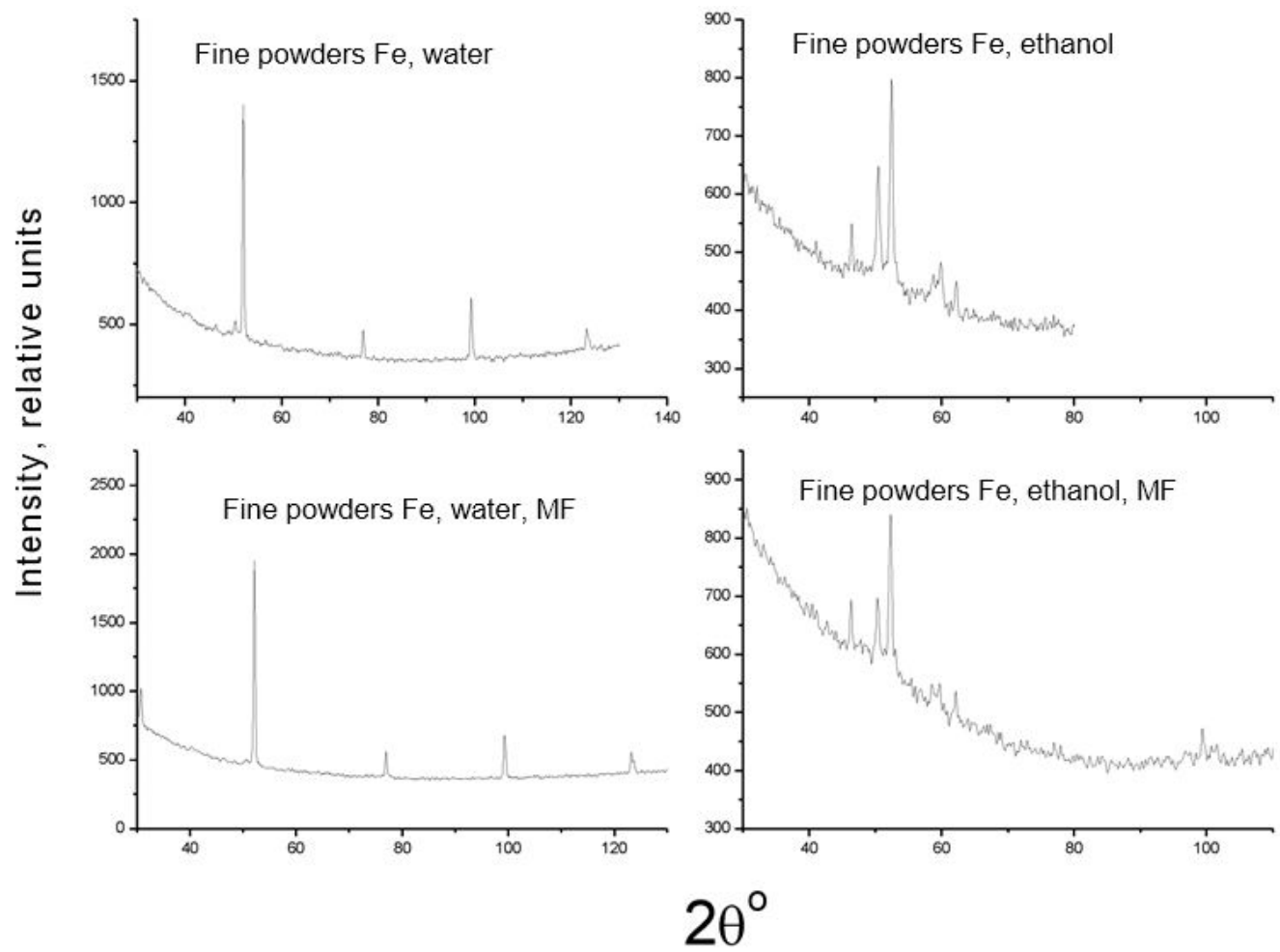

Figure 5: Fragments of diffraction patterns of iron nanoparticles obtained in glycerol and toluene without MF and when it is applied 
Even more significant changes occur when a magnetic field (MF) is applied for the field dependences of the specific magnetizations shown in Figures 6, 7,8 and 9. The first conclusion that can be made when analyzing these dependencies is that the field dependences also react differently to the application of a magnetic field for different liquids. Therefore, for iron nanoparticles obtained in water, the course of the field dependences of the specific magnetization practically does not change when an MF is applied. For nanoparticles of iron, obtained in ethanol and glycerin, the field dependences of the specific magnetization upon the imposition of MPs are higher than the analogous dependences measured without the application of MF, and for nanoparticles of iron, obtained in toluene, on the contrary, lower.

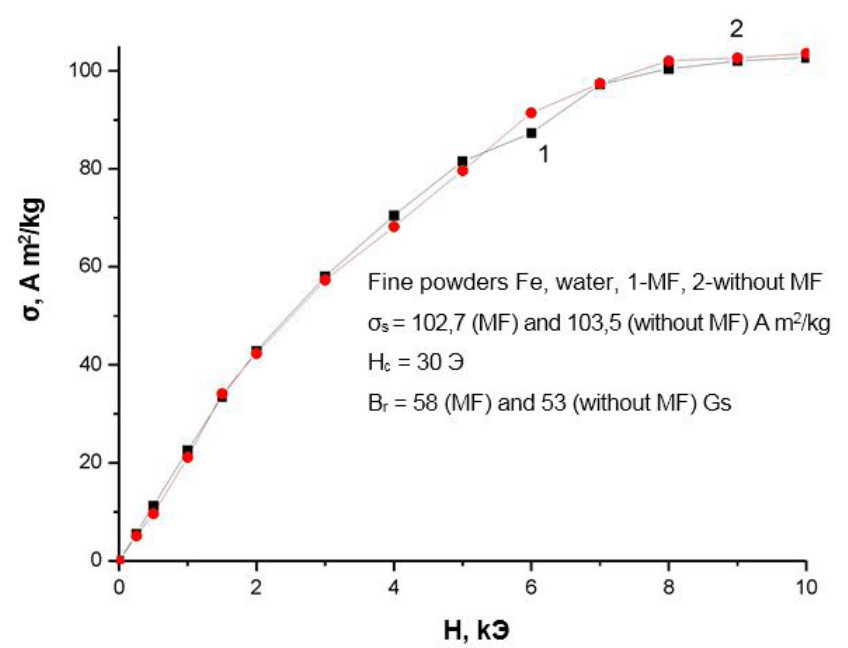

Figure 6: Field dependences of the specific magnetization of iron nanoparticles obtained in water in a zero magnetic field and during its application

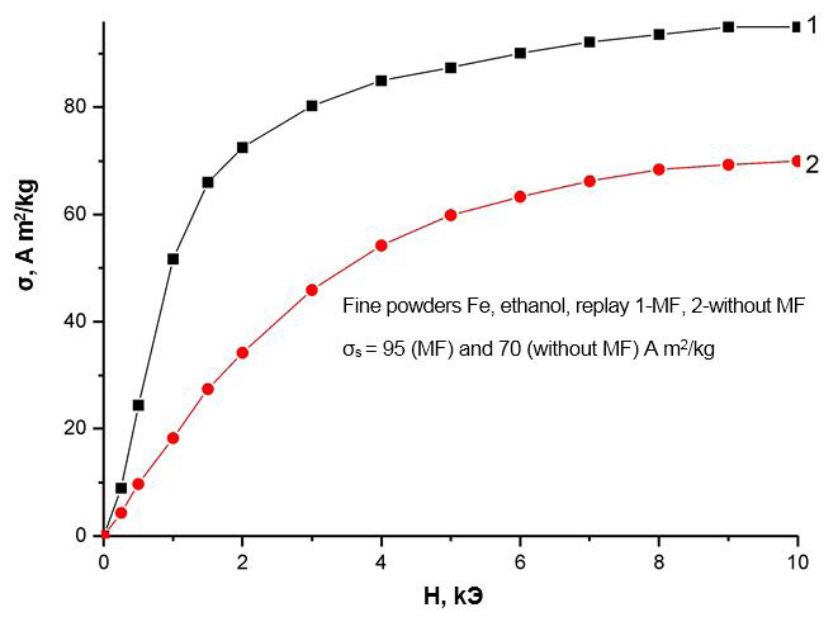

Figure 7: Field dependences of the specific magnetization of iron nanoparticles obtained in ethanol in a zero magnetic field and during its application

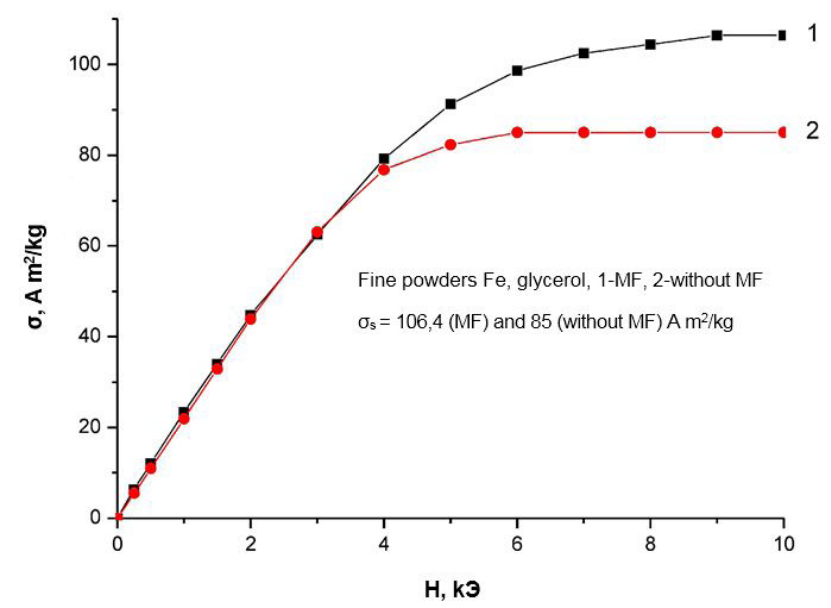

Figure 8: Field dependences of the specific magnetization of iron nanoparticles obtained in glycerol in a zero magnetic field and during its application 


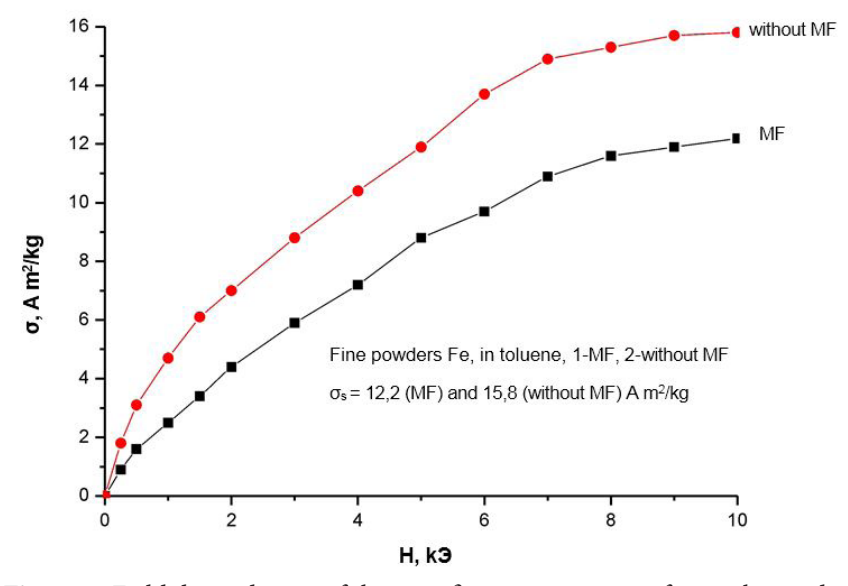

Figure 9: Field dependences of the specific magnetization of iron obtained in toluene in a zero magnetic field and during its application

We now turn to the analysis and discussion of the experimental results obtained. Firstly, an increase in the number of more ferromagnetic $\alpha$ - phases in powders, as well as a decrease in the size of the critical nucleus during the application of MF, can apparently be explained by the negative contribution of the interaction energy of magnetic moments of particles and nuclei of the ferromagnetic phase to the total energy of the system. That leads to the growth of a thermodynamic stimulus for the formation and growth of these embryos. Indeed, when an $M F$ is switched on with a voltage $H$, a particle with a magnetic moment $M$ acquires energy $-\mathrm{M} \times \mathrm{H} \cos \varphi$ (where $\varphi$ is the angle between the direction of the magnetic moment of particle and the applied magnetic field), and now the overall balance of free energy $\Delta \mathrm{F}$ during the formation of a spherical nucleus a ferromagnetic phase with radius $\mathrm{r}$ will look as follows $[37,38]$ :

$$
\begin{aligned}
& \Delta F=-\left(\frac{4}{3}\right) \pi r^{3} \Delta F_{v}+4 \pi r^{2} \gamma_{s}-M H \cos \phi= \\
& =-\left(\frac{4}{3}\right) \pi r^{3}\left(\Delta F_{v}+I s \cdot H \cos \phi\right)+4 \pi r^{2} \gamma_{s},
\end{aligned}
$$

Where $\Delta \mathrm{Fv}$ - change of free energy due to the formation of a spherical nucleus of a new phase in zero MF, $\gamma_{\mathrm{s}}-$ specific surface energy, $I_{S}$ - saturation magnetization of the embryo material. This leads to the fact that in the expression for the radius of the nucleus of critical sizes rc, an additional term appears:

$$
r_{s}=2 \gamma_{s} /\left(\Delta F_{v}+I \cdot H \cos \phi\right)
$$

That should lead to its decrease. This is the picture observed in our experiments. Indeed, the results of measuring the particle size of the smallest fraction, given in Table 1, testify to their decrease with the inclusion of MF.

The same reason can be explained by an increase in the amount of the strongly magnetic a-phase when an MF is applied. It can be shown that the additional term that arises in formula (1) due to the inclusion of MFs will also appear in the expression for the kinetics of nuclei growth of a strongly magnetic phase, which ultimately increases its total amount in the sample.

It should be noted, however, that a change in the phase composition of nanoparticles when an MF is applied cannot explain the observed change in all magnetic characteristics. For example, the imposition of MF in obtaining iron nanoparticles in ethanol leads to an increase in the amount of $\alpha$-phase by $2 \%$, while the specific saturation magnetization increases by almost $30 \%$ (Table 1 and Figure 7). A similar discrepancy is also observed for nanoparticles obtained in glycerol and toluene. On the other hand, the application of MFs in obtaining VDP in distilled water increases the amount of the $\alpha$-phase in the nanoparticle by\% by $1 \%$, although this does not affect the magnitude of the specific saturation magnetization (Figure 6). Additionally, the imposition of MF in obtaining nanoparticles in toluene leads to the opposite compared with nanoparticles obtained in ethanol and glycerin. The effect is that the specific saturation magnetization with the imposition of MF in this case does not increase, but decreases. This decrease can be partially explained by a corresponding $(\sim$ by $2 \%)$ decrease in the amount of the strongly magnetic a-phase. However, this decrease in the amount of phase does not fully explain the magnitude of the observed effect ( 25\%, Figure 9), and, moreover, does not agree with the above arguments.

\section{Conclusions}

In conclusion, it should be noted that the magnitudes of the observed effects could probably be more significant when using external MFs of greater intensity. And yet, studies have shown that the use of external MFs can be used to refine mechanisms for formation of fine particles with DLF materials, and also as an additional factor for controlling the phase composition and dispersion of the powders obtained. 
In conclusion, it should be noted that the magnitudes of the observed effects could probably be more significant when using external MFs of greater intensity. And yet, studies have shown that the use of external MFs can be used to refine mechanisms for formation of fine particles with DLF materials, and also as an additional factor for controlling the phase composition and dispersion of the powders obtained.

\section{References}

1. AD Pomogailo, VN Kestelman (2005) Metallopolymer nanocomposites.

2. DL Leslie Pelecky, RD Rieke (1996) Magnetic Properties of Nanostructures Materials. Chem Mater 8: 1770-83.

3. Raymond JF, Revol DH, Ryan RH, Marchessault (1996) Precipitation of Ferrites in Nafion(R) Membranes. J Appl Polym Sci 59: 1073-86.

4. RVPM Shafi, A Ulman, X Yan, NL Yang, C Estournes, et al. (2001) Sonochemical Synthesis of Functionalized Amorphous Iron Oxide Nanoparticles. Langmuir 17: 5093-7.

5. T Ji, H Shi, J Zhao, Y Zhao (2000) Synthesis of Co-B/resin nanoparticles and heat treatment effect on their magnetic properties. J Magn Magn Mater 212: 189-94. 6. J Ramos, A Millan, F Palacio (2000) Production of magnetic nanoparticles in a polyvinylpyridine matrix. Polymer 41: 8461-4.

7. DL Leslie Pelesky, XQ Zhang, RD Ricke (1996) Self-stabilized magnetic colloids: Ultrafine Co particles in polymers. J Appl Phys 79: 5312-4.

8. SN Sidorov, LM Bronstein, VA Davankov, MP Tsyurupa, SP Solodovnikov, et al. (1999) Cobalt Nanoparticle Formation in the Pores of Hyper-Cross-Linked Polystyrene: Control of Nanoparticle Growth and Morphology. Chem Mater 11:3210-5.

9. RF Ziolo, EP Gianneis, B Weinstein, MP OHoro, BN Ganguly, et al. (1992) Matrix-Mediated Synthesis of Nanocrystalline $\gamma$-Fe2O3: A New Optically Transparent Magnetic Material. Science 257: 219-23.

10. MK Leonowicz, M Lawecka, A wlawska Waniewska GI Dzhardimalieva, AS Rozenberg, et al. (2003) Magnetic properties of a polymer matrix nanocomposites on a basis of metal carboxylates. Macromol Symp 204: 257-66.

11. M Ławecka, M Kopcewicz, A lawska Waniewska, J Kozubowski, M Leonowicz, et al. (2003) Formation, structured by the thermal decomposition of the Fe (III) Co (II) acrylate complex. J Nanocomposite Research 5: 373-81.

12. M Leonowicz, M Ławecka, GI Dzhardimalieva, DN Muraviev, AS Rozenberg, et al. (2003) Magnetic Properties of Polymer Matrix Nanocomposites on a Basis of Metal Carboxylates. Proc. 10th IUPAC International Symposium on Macromolecule-Metal Complexes 204: 257-66.

13. M Ławecka, A lawska Waniewska, M Leonowicz, GI Dzhardimalieva, AS Rozenberg, et al. (2004) Structure and magnetic properties of polymer matrix nanocomposite processed by pyrolysis of cobalt(II) acrylate. J Alloys and Compounds 369: 244-6.

14. M Leonowicz, M Woźniak, YM Shulga, Z Liu, HA Davies, et al. (2005) Structure and magnetic properties of nanoparticles encapsulated in carbon shells. J Magn Magn Mater 294: 57-62.

15. M Leonowicz, M Woźniak, YM Shulga, VE Muradyan, Z Liu, et al. (2006) Processing and properties of magnetic nanoparticles encapsulated in carbon shells. Materials Letters 60: 442-6.

16. J Gómez Morales, J García Carmona, R Rodríguez Clemente, D Muraviev (2003) Preparation of Sub-Micro Chromium Hydroxide and Nanoparticles Microwave Dielectric Heating. Langmuir 19: 9110-3.

17. SP Gubin (2002) Metalcontaining nanoparticles within polymeric matrices: preparation, structure and properties. Colloids and Surfaces A: Physicochem. Eng. Aspects 202: 155-63.

18. S Sun, C Murray, D Weller, L Folks, A Moser (2000) Monodisperse FePt Nanoparticles and Ferromagnetic FePt Nanocrystal Superlattices. Science 287: 1989-92. 19. V Skumryev, S Stoyanov, Yong Zhang, G Hadjipanayis, D Givord, et al. (2003) Beating the superparamagnetic limit with exchange bias. Nature 423: 850-3.

20. KV Chuistov, AP Shpak, AE Perekos, OD Rud, VM Uvarov (2003) Small Metallic Particles: Methods of Fabrication, Atomic and Electronic Structure, Magnetic Properties, and Practical Usage. UFM 4: 235-69.

21. Veziroglu TN, Zaginaichenko SY, DV Schur, AG Dubovoy, S Yu, Zaginaichenko et al. (2007) Synthesis Of Carbon Nanostructures In Gaseous And Liquid Medium Hydrogen Materials Science and Chemistry of Carbon Nanomaterials: 199-212.

22. SS Gorelik, Skakov YA, Rastorguev LN (1994) Radiographic and Electron Optical Analysis.

23. AE Berkowitz, JL Walter (1987) Spark erosion: A method for producing rapidly quenched fine powders. J Mater Res 2: 277-88.

24. AG Dubovoy, VP Zalutsky, I Yu Ignatieva, AE Perekos (1986) Metallophysics 86: 101.

25. G Monastyrsky, AP Shpak, Yu M Koval, AA Shcherba (2003) Metallofiz the newest technol 25: 803.

26. VI Trefilov, DV Schur, BP Tarasov, Yu M Shulga, AV Chernogorenko, et al. (2001) Zaginaychenko, Fullerenes - the basis of the materials of the future, Monograph 148.

27. BP Tarasov, VE Muradyan, Yu M Shulga, EP Krinichnaya, NS Kuyunko, et al. (2003) Synthesis of carbon nanostructures by arc evaporation of graphite rods with Co-Ni and YNi2 catalysts. Carbon 41: 1357-64.

28. AA Rogozinskaya, DV Schur, II Timofeeva, A Klochkov, AP Simanovskiy, et al. (2004) NATO Science Series II - Mathematics, Physics and Chemistry 172: 307. 29. Yu M Shulga, DV Schur, SA Baskakov, AP Simanovskiy, AA Rogozinskaya, et al. (2004) NATO Science Series II - Mathematics, Physics and Chemistry 172: 137. 30. S Yu Zaginaichenko, DV Schur, ZA Matysina (2003) The peculiarities of carbon interaction with catalysts during the synthesis of carbon nanomaterials. Carbon 41: 1349-55.

31. AD Zolotarenko, AF Savenko, AN Antropov, MI Maystranko, RN Nikalenko (2003) Proceedings of the NATO Advanced Research Workshop on Carbon Nanomaterials, Sudak, Crimea, Ukraine 172: 217.

32. DV Schur, SY Zaginaichenko, AD Zolotarenko, TN Veziroglu (2008) Carbon Nanomaterials in Clean Energy Hydrogen Systems. NATO Science Series 85. 33. DV Schur, AA Liashenko, VM Adejev, VB Voitovich, SY Zaginaichenko (1995) Niobium as a construction material for a hydrogen energy system. International Journal of Hydrogen Energy 20: 405-7. 
34. MD Hampton, DV Schur, SY Zaginaichenko, VI Trefilov (2002) Hydrogen Materials Science and Chemistry of Metal Hydrides. Springer Science \& Business Media 71: 586.

35. SY Zaginaichenko, DV Schur, ZA Matysina (2003) The peculiarities of carbon interaction with catalysts during the synthesis of carbon nanomaterials. Carbon 41: 1349-55.

36. SY Zaginaichenko, ZA Matysina, DV Schur (1996) The influence of nitrogen, oxygen, carbon, boron, silicon and phosphorus on hydrogen solubility in crystals. Int J Hydrogen Energy 21: 1073.

37. KV Chuistov (2003) Aging of metal alloys.

38. Ya S Umansky, Yu A Skakov (1978) Metal Physics (Moscow: Atomizdat). 